\title{
GPDs At HERMES
}

\author{
Aram Movsisyan*i \\ Yerevan Physics Institute \\ E-mail: aram.movsisyanedesy.de
}

The formalism of Generalized Parton Distributions (GPDs) is currently considered to be one of the most promising frameworks to provide a three dimensional description of the nucleon structure. The interest to GPDs is also governed by their relation to the quark angular momentum in the nucleon. Experimentally, the GPDs can be accessed through measurements of hard exclusive processes such as hard leptoproduction of real photons or mesons. The HERMES experiment at DESY Hamburg studies hard exclusive processes using polarized electron or positron beams from HERA and internal gas targets. Information about GPDs is gained from the measurements of asymmetries that appear in the azimuthal distributions of produced mesons and photons.

Sixth International Conference on Quarks and Nuclear Physics,

April 16-20, 2012

Ecole Polytechnique, Palaiseau, Paris

*Speaker.

$\dagger$ on behalf of the HERMES Collaboration 


\section{Introduction}

The formalism of Generalized Parton Distributions (GPDs) [1]-3] is nowadays a commonly used framework for the description of nucleon structure in terms of quark and gluon degrees of freedom. Traditionally, the structure of the nucleon was described by elastic form factors, representing a transverse spatial distributions of charge and magnetization in the nucleon, and by parton distribution functions, representing a distributions of longitudinal momentum fractions of partons in the nucleon. An experimental information about form factors is obtained from elastic scattering experiments, while the parton distribution functions are measured in inclusive or semi-inclusive deep inelastic scattering experiments. In contrast to form factors and parton distribution functions, which are one dimensional distributions, the GPDs contain a correlated information on transverse spatial and longitudinal momentum distributions of partons, thus providing a more comprehensive, multidimensional description of the nucleon [甘, 印. As a consequence, the form factors and parton distribution functions appear as limiting cases and moments of GPDs, respectively. Another interesting feature of GPDs is their sensitivity to the total angular momentum of partons in the nucleon according to Ji relation [3].

Generalized parton distributions depend upon four kinematic variables: the Mandelstam variable $t=\left(p-p^{\prime}\right)^{2}$, which is the squared momentum transfer to the nucleon with $p$ and $p /$ representing the initial and final four-momentum of the nucleon; the average fraction $x$ of the nucleon's longitudinal momentum carried by the active parton under the consideration; half the difference of the fractions of the nucleon's longitudinal momentum carried by the active parton at the initial and final state, written as the skewness $\xi$; and $Q^{2}$, i.e., the negative square of the four-momentum of the virtual photon that mediates the lepton-nucleon scattering process.

Experimentally, the GPDs can be accessed trough measurements of hard exclusive leptonnucleon scattering processes, such as hard leptoproduction of real photons or mesons. Variety of exclusive processes were investigated at HERMES using a longitudinally polarized $27.6 \mathrm{GeV}$ lepton beam with both charges and both helicities and longitudinally/transversally polarized hydrogen target. The hard exclusive processes on spin-1/2 nucleon are described by four leading-twist GPDs $(H, E, \widetilde{H}$, and $\widetilde{E})$, while certain experimental observables require a consideration of higher-twist contributions as well.

\section{Deeply Virtual Compton Scattering}

Deeply Virtual Compton Scattering (DVCS), the hard exclusive production of real photons where the photon is emitted by a struck quark in a proton, is currently the cleanest process that provides an access to GPDs. Beside DVCS, there is another process with the same initial and final state, Bethe-Heitler (BH), where the final photon is radiated by an incoming or outgoing lepton. These processes are experimentally indistinguishable, and therefore the total cross section of hard leptoproduction of real photons contains an interference term $(\mathscr{I})$, which depends on the charge of the lepton beam.

$$
d \sigma \propto\left|\mathscr{T}_{B H}\right|^{2}+\left|\mathscr{T}_{D V C S}\right|^{2}+\mathscr{I} .
$$

The individual terms from the cross section can be decomposed into Furier harmonics in azimuthal angle $\phi$, which is defined as an angle between the lepton scattering plane and the photon 
production plane. Different Furier components depend on different Compton Form Factors (CFF), which in turn are convolutions of hard scattering amplitudes with the corresponding GPDs [6].

At HERMES, the DVCS process is accessed through measurements of cross section asymmetries that appear in the azimuthal distributions of final state photons. Utilizing data collected with longitudinally polarized electron/positron beam with both target polarization states, it is possible to measure asymmetries with respect to beam charge, beam polarization and target polarization alone and also with respect to their different combinations. As an example, the asymmetries in the cross section for scattering a longitudinally polarized electron/positron beam off a transversely polarized hydrogen target can be described as

$$
\begin{aligned}
d \sigma & =d \sigma_{U U}(\phi)\left[1+e_{\ell} A_{C}(\phi)+P_{\ell} A_{L U}^{D V C S}(\phi)+S_{\perp} A_{U T}^{D V C S}\left(\phi, \phi_{S}\right)+e_{\ell} P_{\ell} A_{L U}^{I}(\phi)\right. \\
& \left.+e_{\ell} S_{\perp} A_{U T}^{I}\left(\phi, \phi_{S}\right)+P_{\ell} S_{\perp} A_{L T}^{B H+D V C S}\left(\phi, \phi_{S}\right)+e_{\ell} P_{\ell} S_{\perp} A_{L T}^{I}\left(\phi, \phi_{S}\right)\right] .
\end{aligned}
$$

Here $d \sigma_{U U}$ is the cross section of scattering an unpolarized lepton beam off an unpolarized target averaged over both beam charges, $e_{\ell}$ and $P_{\ell}$ are the beam charge and polarization respectively, and the $S_{\perp}$ is the transverse target polarization. The angle $\phi_{S}$ is defined as the angle between the lepton scattering plane and the transverse component of the target polarization. For the case of longitudinally polarized hydrogen or deuterium target, it is possible to measure another set of asymmetries with respect to the longitudinal polarization of the target. The asymmetries from Eq. 2.2 were expanded into Fourier harmonics in the azimuthal angles $\phi$ and $\phi_{S}$ and the corresponding asymmetry amplitudes were extracted simultaneously using a maximum likelihood fit.

An overview of all extracted azimuthal asymmetry amplitudes corresponding to the entire HERMES kinematics is presented in Figure 1 for both Hydrogen and Deuterium targets $17-$ 9. The amplitudes of the beamhelicity and beam-charge asymmetries $A_{L U}^{D V C S}(\phi), A_{L U}^{I}(\phi)$ and $A_{C}(\phi)$ are presented in the top panels of Figure 1. A

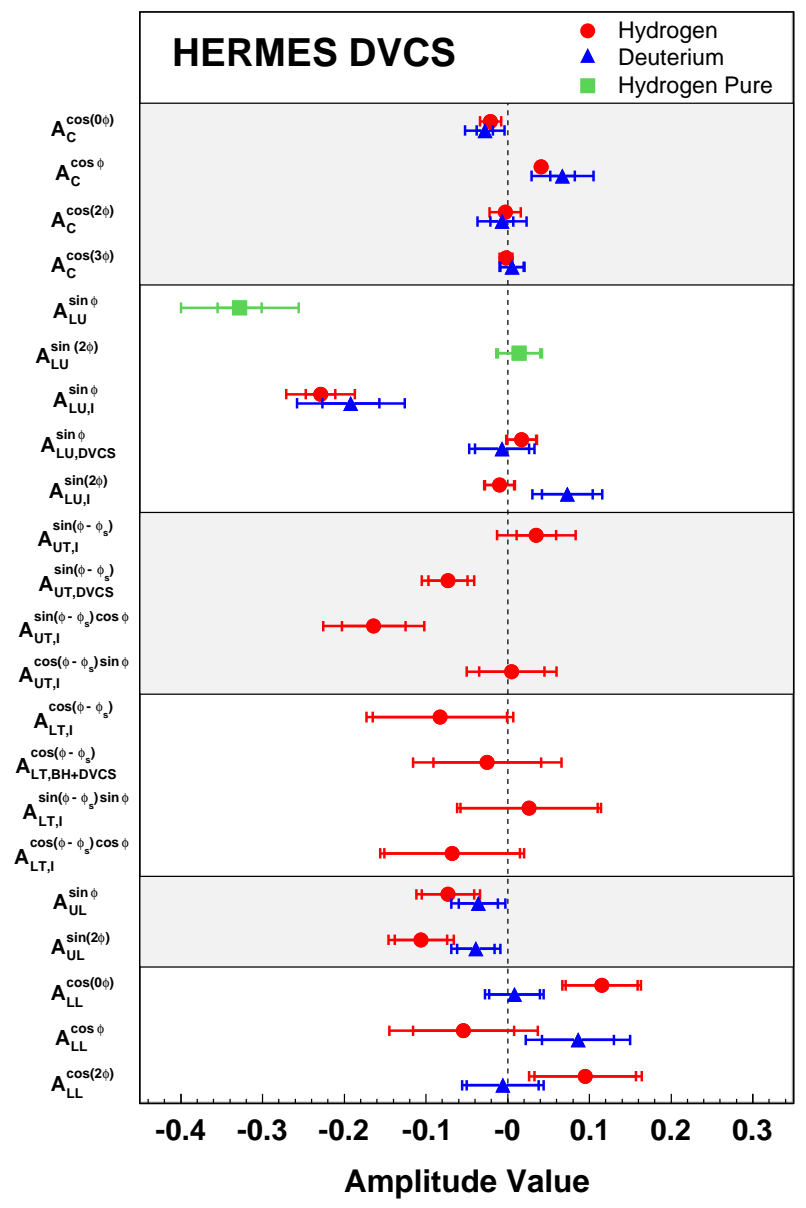

Figure 1: Overview of azimuthal asymmetries extracted at HERMES. 
significant non-zero $\cos (\phi)$ and $\sin (\phi)$ amplitudes were observed respectively for beam-charge $A_{C}(\phi)$ and beam-helicity $A_{L U}^{I}(\phi)$ asymmetries. These two amplitudes are sensitive to the real and imaginary parts of CFF $\mathscr{H}$, respectively. The results for $\sin (\phi)$ amplitude of the charge-averaged beam-helicity asymmetry $A_{L U}^{D V C S}(\phi)$ are consistent with zero. In the bottom panels of Figure 1, the results of the longitudinal single-target-spin $A_{U L}(\phi)$ and double-spin $A_{L L}(\phi)$ asymmetries are presented. For the case of hydrogen target, the leading amplitudes of these asymmetries are sensitive to the imaginary and real parts of CFF $\widetilde{\mathscr{H}}$, respectively. Also shown in Figure 1 are the leading amplitudes of transverse single-target-spin and double-spin asymmetries, measured on Hydrogen target. The amplitude $A_{U T, I}^{\sin \left(\phi-\phi_{S}\right) \cos (\phi)}$, which has significant negative value, is sensitive to the imaginary part of CFF $\mathscr{E}$, while the amplitude $A_{L T, I}^{\sin \left(\phi-\phi_{S}\right) \sin (\phi)}$, which is consistent with zero, is sensitive to the real part of CFF $\mathscr{E}$.

The above mentioned results for the asymmetry amplitudes were obtained without recoil proton detection. The exclusivity of the process was achieved requiring the missing mass, calculated from lepton and photon kinematics, to be close to the proton mass. This method does not allow the separation of the pure DVCS/BH events from that of "associated" process where the proton excites to the baryonic resonant state. The associated background contributes about $12 \%$ in the measured exclusive sample.

For the data collected with the recoil proton detection, it is possible to exclude the contribution of associated background and achieve purity of DVCS/BH process of more than $99 \%$. The results of the single-charge beam-helicity asymmetry amplitudes $A_{L U}^{\sin (n \phi)}$ from the pure sample are also presented in Figure 1. In order to compare results under similar kinematic conditions, a "reference" event sample was created where in addition to the selection criteria used for the unresolved sample a "hypothetical" proton with 3-momenta calculated from the 3-momenta of electron and photon was required to be in the recoil detector acceptance. For this subsample, background conditions are very similar to those for the unresolved sample. Hence, it can be used for comparison with the background-free measurement. The results for unresolved, unresolved reference and pure samples are compared in Figure .

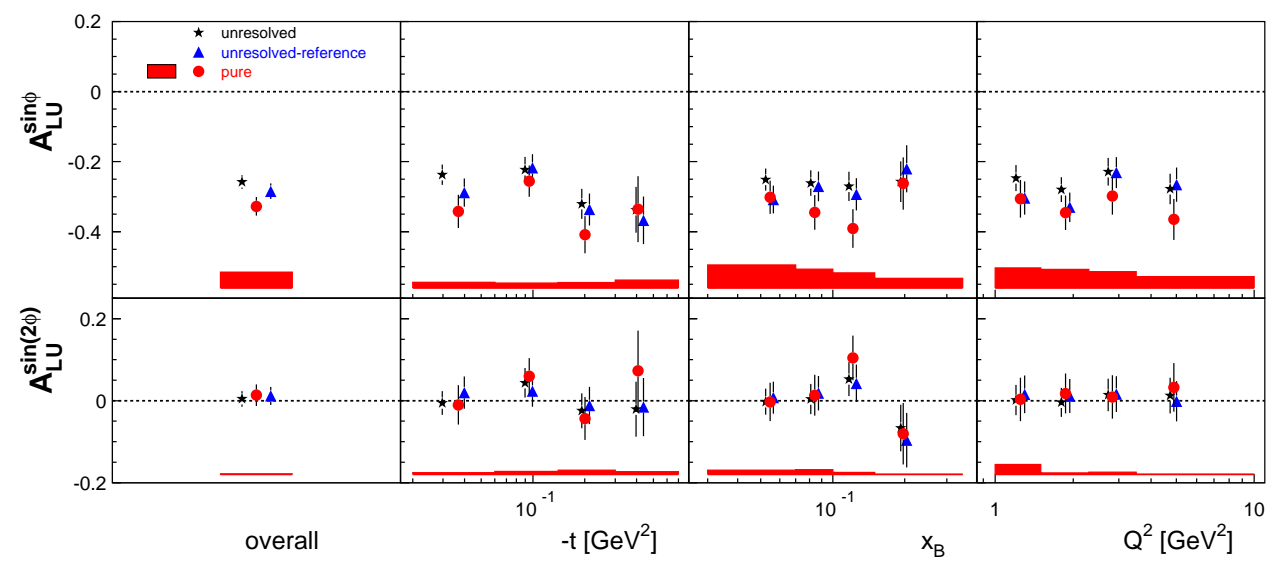

Figure 2: Amplitudes of single-charge beam-helicity asymmetry $A_{L U}$ extracted from unresolved, unresolved reference and pure samples. 


\section{Hard Exclusive Meson Production}

The investigation of hard exclusive meson production provides an independent set of processes to study GPDs. There are two alternative approaches for the theoretical description of exclusive meson production. First approach relies on the Regge phenomenology, while the second approach is based on perturbative QCD (pQCD) description of the processes in terms of handbag diagrams. In the later case, the GPDs are involved in the parametrization of non-perturbative part of the diagrams. In the $\mathrm{pQCD}$ approach, the virtual photon dissociates into $q \bar{q}$ pair, whose interaction with nucleon results in formation of a meson. The interaction can proceed via two distinct mechanisms: quark-antiquark or two-gluon exchange, therefore providing an information also

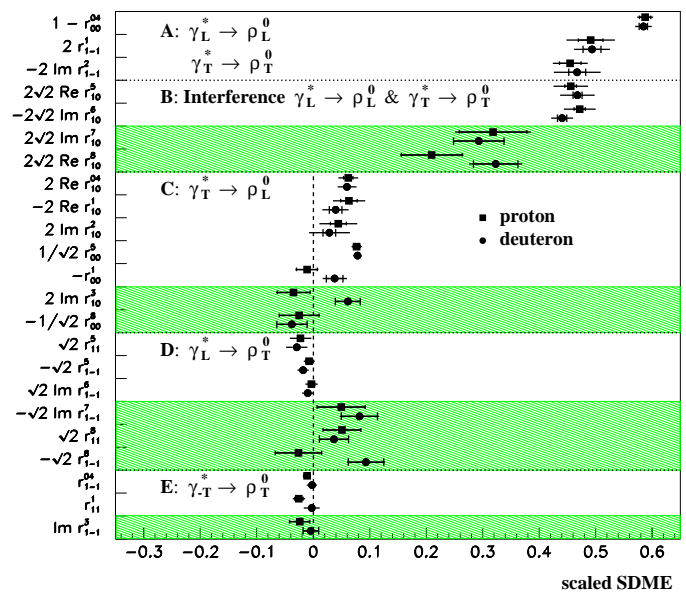

Figure 3: $\rho^{0}$ SDMEs. about gluon GPDs. In the case of exclusive pseudo-scalar meson production, the experimental observables are sensitive to GPDs $\widetilde{H}$ and $\widetilde{E}$, while in the case of exclusive vector meson production the GPDs $H$ and $E$ can be accessed.

The spin transfer from the virtual pho-

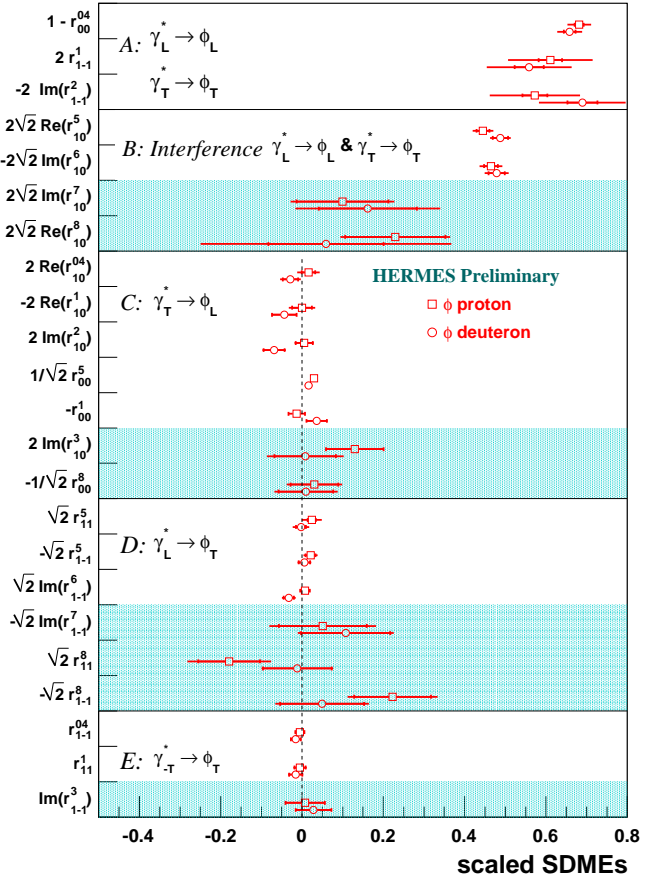

Figure 4: $\phi$ SDMEs. ton $\gamma^{*}$ to the vector meson is commonly described in terms of Spin Density Matrix Elements (SDMEs) [10], which in turn are related to different helicity amplitudes. The scattering of longitudinally polarized leptons off an unpolarized targets is described by 23 SDMEs, which can be obtained from decay angular distributions in the vector meson production. The SDMEs in exclusive $\rho^{0}$ production corresponding to the entire HERMES kinematics are presented in Figure 3 measured on both Hydrogen and Deuterium targets [11]. They are grouped into different classes corresponding to different spin transitions between virtual photon and vector meson. The SDMEs measured with unpolarized (polarized) beam are displayed in the unshaded (shaded) areas. Preliminary results of $\phi$ SDMEs are presented in Figure 4 . The measured SDMEs of classes $\mathrm{A}$ and $\mathrm{B}$ have significant non-zero values for both $\rho^{0}$ and $\phi$ mesons. There is in average $10-20 \%$ difference between results for $\rho^{0}$ and $\phi$ 


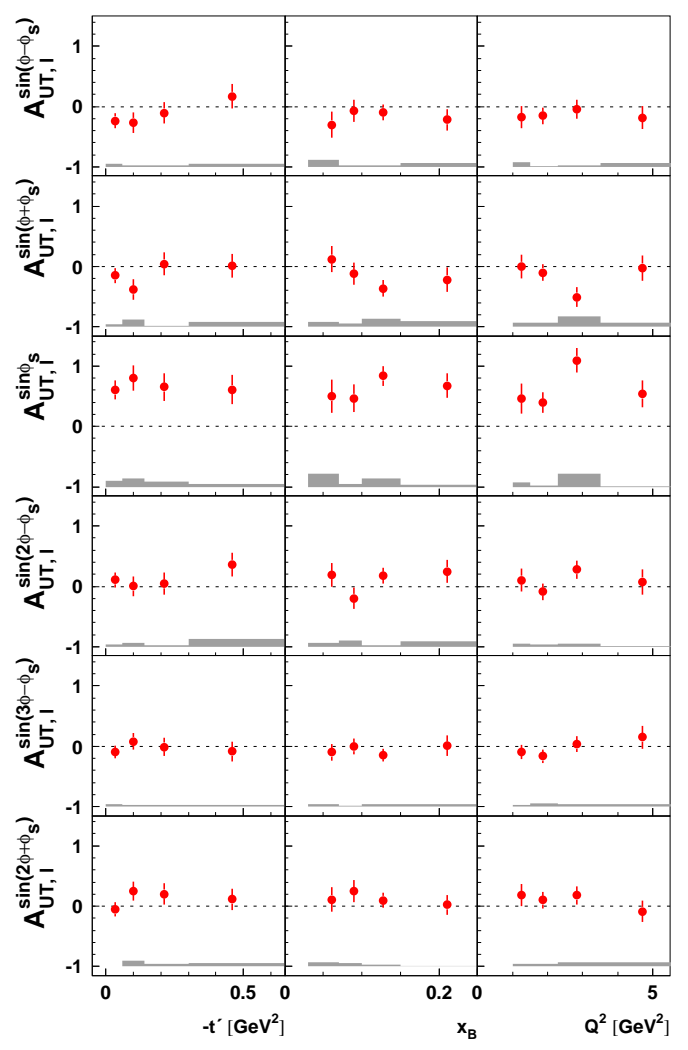

Figure 5: Amplitudes of $A_{U T}\left(\phi, \phi_{S}\right)$ asymmetry in exclusive $\pi^{+}$production. meson productions. A significant non-zero values of SDMEs of class $C$ are observed for $\rho^{0}$ meson. This indicates that their is a production mechanism that does not conserve s-channel helicity. The $\phi$ meson SDMEs of the same class are consistent with zero. The SDMEs of classes D and E are also consistent with zero for both mesons except from the polarized $\rho^{0}$ SDMEs, which show slightly positive values. No significant differences are observed between SDMEs measured on Hydrogen and Deuterium targets.

Figure 5 shows measured results of transverse target-spin asymmetry amplitudes in exclusive $\pi^{+}$production as a function of $-t$, $x_{B}$ and $Q^{2}$ [12]. Of special interest is the amplitude $A_{U T}^{\sin \left(\phi-\phi_{S}\right)}$, which is sensitive to GPDs $\widetilde{H}$ and $\widetilde{E}$. This amplitude was found to be consistent with zero, suggesting a possible dominance of GPD $\widetilde{E}$ over $\widetilde{H}$. The rest of the amplitudes are compatible with zero except $A_{U T}^{\sin \left(\phi_{S}\right)}$ indicating a significant contribution from the transverse-tolongitudinal helicity transition of the virtual photon.

\section{References}

[1] D. Muller, et al., Fortsch. Phys. 42101 (1994).

[2] A.V. Radyushkin, Phys. Lett B380 417 (1996), Phys. Rev. D56 5524 (1997).

[3] X. Ji, Phys. Rev. Lett. 78610 (1997), Phys. Rev. D55 7114 (1997).

[4] J.P. Ralston, B. Pire Phys. Rev. D66 111501 (2002).

[5] M. Burkardt, Phys. Rev. D62 071503 (2000); Erratum-ibid C31 277 (2003).

[6] A.V. Belitsky, D. Muller, A. Kirchner, Nucl. Phys. B629 323 (2002).

[7] A. Airapetian et al.,[HERMES Collab.], JHEP 07032 (2012), JHEP 06019 (2010), JHEP 06066 (2008), Phys. Lett. B 70415 (2011).

[8] A. Airapetian et al.,[HERMES Collab.], Nucl. Phys. B829 1 (2010), Nucl. Phys. B842 265 (2011).

[9] A. Airapetian et al.,[HERMES Collab.], arXiv:1206.5683 [hep-ex].

[10] K. Schillimng, G. Wolf, Nucl. Phys. B61 381 (1973).

[11] A. Airapetian et al.,[HERMES Collab.], EPJC 62659 (2009).

[12] A. Airapetian et al.,[HERMES Collab.], Phys. Lett. B 682345 (2010). 